\title{
Wheezing, CTCAE
}

National Cancer Institute

\section{Source}

National Cancer Institute. Wheezing, CT CAE. NCI Thesaurus. Code C143947.

A disorder characterized by a high-pitched, whistling sound during breathing. It results from the narrowing or obstruction of the respiratory airways. 